\title{
The Dynamic Behaviour of a Twinning Induced Plasticity Steel
}

\author{
K.M. Rahman ${ }^{\mathrm{a}}$, V.A. Vorontsov ${ }^{\mathrm{a}}$, D. Dye $\mathrm{D}^{\mathrm{a},, 1}$ \\ ${ }^{a}$ Department of Materials, Royal School of Mines, Imperial College London, Prince Consort Road, London SW7 2BP, UK
}

\begin{abstract}
The influence of strain rate on the twinning behaviour and microstructure of an Fe-15Mn-2Al-2Si-0.7C twinning induced plasticity (TWIP) steel has been investigated. A Hopkinson pressure bar setup was used in addition to blast testing to perform the high strain rate testing. The yield stress exhibited a positive strain rate sensitivity with increasing strain rate. However, the failure strain of the material was relatively unaffected. Post deformation microscopy indicated that deformation twinning was less profuse at higher strain rates. Electron backscatter diffraction also indicated the activation of multiple twin systems at strain rates below $1000 \mathrm{~s}^{-1}$ although this did not occur at the higher strain rates tested. A large intragranular misorientation was found to exist in the material tested at lower strain rates indicating a relatively larger dislocation density existing in the material tested at lower strain rates. In addition selected grains in the blast tested material exhibited a 'wavy' structure which was determined not to be due to a phase transformation. It is suggested that this was caused by the complex loading experienced by the material during testing. High resolution transmission electron microscopy also indicated a large density of intrinsic stacking faults in the material subjected to blast testing.
\end{abstract}

Key words: Twinning, Mechanical Characterisation, Austenite, Yield Phenomena, Steel

\section{Introduction}

The pursuit of steels with a combination of high strength and excellent formability has been a driving force for the ${ }_{30}$ continous development and evolution of new alloys within ${ }_{31}$ the steel industry. This requirement has contributed to the development of TWinning Induced Plasticiy (TWIP) steels. These steels posses an excellent combination of ${ }_{34}$ strength and ductility which can be as high as $800 \mathrm{MPa}_{35}$ and $95 \%$ respectively $[1,2]$. These exceptional mechan- ${ }_{36}$ ical properties are obtained from a high work hardening ${ }_{37}$ capacity within the material which is attributed to the ${ }_{38}$ continuous formation of mechanical twins during deformation. Hence, these properties make the alloys ideal candi- ${ }_{40}$ date materials for energy absorption applications, includ- ${ }_{41}$ ing military vehicle armour and automotive crash safety.

The TWIP mechanism occurs in stable austenite in ${ }_{43}$ alloys which have an intermediate stacking fault energy ${ }_{44}$ (SFE) that is generally between $18-45 \mathrm{~mJ} \mathrm{~m}^{-2}$. A SFE ${ }_{45}$ below this range favours a strain induced phase transformation to $\varepsilon$-martensite while a higher SFE favours defor- ${ }_{47}$ mation to proceed solely via a dislocation glide mecha- ${ }_{48}$ nism [2-4]. To fully utilise the twinning mechanism the ${ }_{49}$ chemical composition of the alloy has to be adjusted to ${ }_{50}$ maintain the required SFE range. Therefore, TWIP steels ${ }_{51}$ are alloyed with high levels of manganese with the addi- ${ }_{52}$ tion, in most cases, of silicon and aluminium. These three ${ }_{53}$

Email addresses: david.dye@imperial.ac.uk (D. Dye)

${ }^{1} \mathrm{Tel}:$ +44 2075946811 elements act as austenite stabilisers [5]. In addition, manganese and aluminium act to raise the SFE while silicon lowers it $[6-8]$.

TWIP steels are characterised by the formation of mechanical twins during deformation. These twins produce a dynamic Hall-Petch effect within the material and it is believed that this generates the impressive strain hardening exhibited in TWIP steels [5, 9]. During deformation, twins are nucleated and subsequently act as obstacles for gliding dislocations. This effectively results in a continuous grain refinement process, leading to a reduction in the dislocation mean free path and producing the characteristic high hardening rate observed. Several mechanisms for twin nucleation and growth have been proposed. However, it is generally accepted that the formation of deformation twins proceeds through a dislocation mechanism, whether by a pole mechanism [10], a deviation process, i.e. through the production of intrinsic stacking faults along with the nucleation of sessile Frank dislocations [11] or by twin nucleation through the formation of stacking faults [12].

The strain rate sensitivity of a material is an important property and requires significant attention when considering dynamic loading applications such as vehicle crush or armour protection systems. These applications are where high strain rate deformation is highly likely to occur. A material which exhibits a strong positive strain rate sensitivity is therefore ideal for these applications. This implies that the faster a load is applied the more readily the material will resist deformation. Materials that deform solely 
though a slip mechanism typically exhibit an increase in 114 strength with increasing strain rates. This is usually ex-115 plained by the theory of thermally activated dislocation 116 motion where the time for a dislocation to wait in front of fit $_{1}$ an obstacle for additional thermal energy is reduced. ${ }_{118}$

The contribution of mechanical twinning during defor-119 mation in f.c.c. metals increases as the temperature is 120 reduced. This is because the twin stress, i.e. the stress 121 required to nucleate a deformation twin, reduces gently 122 with decreasing temperature [12]. The weak dependence ${ }_{123}$ on temperature has led to the twin nucleation stress to124 be considered essentially athermal [13]. However, the twin 125 nucleation stress is also dependent on the SFE in f.c.c.126 metals. The SFE is strongly dependent on temperature $[\mathbf{3}, 127$ 14, 15]. Therefore, the local material temperature will ${ }_{128}$ substantially affect the proceeding deformation mechanism ${ }_{12}$ occurring within the material [5]. The effect of temper-130 ature on the deformation behaviour in metals is closely131 related to strain rate due to adiabatic heating and hasis2 thus typically been coupled using an Arrhenius type re-13з lationship [15]. This also implies that it is a thermally134 activated process [12]. Mahajan et al. [12] have reported ${ }_{135}$ that the contention between slip and twinning has a weak ${ }_{136}$ temperature sensitivity but the twin stress has a very dis-137 tinct strain rate sensitivity. A possible explanation for the negative strain sensitivity of twinning has been proposed by Bolling et al. [16]. Here the authors suggested that the ${ }^{138}$ high stress concentrations at the edge of a twin result in lo- ${ }_{139}$ calised slip in the region for which the theory of thermally activated dislocation motion is valid, and consequently at ${ }^{140}$ higher strain rates this dependency is inverted.

Adiabatic heating due to dynamic loading is considered ${ }^{142}$ to be sufficient to influence the SFE of the metal. Curtze ${ }^{143}$ et al. [3] have shown that during the high strain rate defor- ${ }^{144}$ mation of a TWIP steel at $\sim 1000 \mathrm{~s}^{-1}$ a temperature rise of $\mathrm{f}^{145}$ $\sim 95^{\circ} \mathrm{C}$ can occur within the material. This consequently ${ }^{146}$ leads to a SFE increase of $\sim 25 \mathrm{~mJ} \mathrm{~m}^{-2}$. The authors also concluded that a weak to moderate strain rate sensitivity ${ }^{148}$ is exhibited at strain rates between $10^{-3}-750 \mathrm{~s}^{-1}$ while ${ }^{149}$ a steep upturn in strength is observed at $1000 \mathrm{~s}^{-1}$. Fur- ${ }^{150}$ thermore, at high strain rates, a reduction in elongation ${ }^{151}$ was observed which was explained to be caused by an in ${ }^{152}$ crease in SFE through adiabatic heating which promotes ${ }^{153}$ less twinning. Xiong et al. [17] have similarly observed a significant temperature rise in a silicon and aluminium rich ${ }^{154}$ TWIP steel at strain rates between $700-2500 \mathrm{~s}^{-1}$. How- ${ }^{155}$ ever the authors report that the twin stress reduces and the $\mathrm{e}^{156}$ density of deformation twins increases with higher strain ${ }^{157}$ rates.

In addition to adiabatic heating effects on the SFE, dy_-159 namic recrystallisation has also been observed during high ${ }^{160}$ strain rate testing of TWIP steels. Sahu et al. [18] have ${ }^{161}$ reported on the occurrence of dynamic recrystallisation ${ }^{162}$ caused by adiabatic heating at high strain rates. An in- ${ }^{163}$ crease in austenite stability is also reported via a reduction ${ }^{164}$ in the driving force for the $\varepsilon$-martensite transformation ${ }^{165}$ and therefore increasing the critical twin stress. However, ${ }^{166}$ this observation appears to contradict the theory proposed by Mahajan et al. [12]. Li et al. [19] have reported on localised amorphism after high strain rate ballistic testing of a TWIP steel. Here the authors found adiabatic shear banding to be the main deformation mode in addition to slip and twinning. The authors also reported that some adiabatic shear bands (ASBs) in highly deformed areas exhibited a gradual microstructure change from amorphous, amorphous-crystalline to nanocrystalline $(<10 \mathrm{~nm})$ structures evolving from the centre of the ASBs outwards, thus indicating that localised melting and fast quenching occurred due to the adiabatic heating.

Relatively few studies have been conducted that investigate the dynamic loading behaviour of TWIP steels. The majority of the existing research has been performed at strain rates up to and below $1000 \mathrm{~s}^{-1}$. In the present study, the effect of dynamic loading at a range of strain rates on the mechanical behaviour of a TWIP steel has been examined using Hopkinson pressure bar and blast testing. In addition, a range of microscopy techniques has been used to augment the mechanical testing observations to elucidate the role of strain rate on the characteristics of twinning during deformation when compared to quasistatic loading.

\section{Experimental Procedures}

\subsection{Material}

The TWIP steel used in this investigation had a nominal composition of $15 \mathrm{Mn}-2 \mathrm{Al}-2 \mathrm{Si}-0.7 \mathrm{C}$ wt. \%. The material was supplied in $3 \mathrm{~mm}$ rolled sheet form by Tata Steel Strip Mainland Europe. The average grain size of the material was determined to be $10 \pm 6 \mu \mathrm{m}$ and a random texture was identified using three different experimental techniques, Figure 1. The three sets of pole figures have been post-processed to obtain the pole figures and therefore appear different. However, the texture intensities are low and no obvious texture components can be identified for all three pole figure sets, thus indicating a random texture. The stacking fault energy of the alloy was calculated to be $30 \pm 10 \mathrm{~mJ} \mathrm{~m}^{-2}$ using thermodynamics based models and data $[6,7,20]$.

\subsection{Quasi-Static and Intermediate Strain Rate Testing}

Tensile testing at nominal strain rates of $10^{-3}$ and $10^{-1} \mathrm{~s}^{-1}$ was conducted on a Zwick Roell $100 \mathrm{kN}$ load frame using a $10 \mathrm{~mm}$ gauge length extensometer. The test specimens used had gauge dimensions of $19 \times 1.5 \times 1.5 \mathrm{~mm}$ and were tested aligned to the rolling direction (RD) of the plate.

Intermediate strain rate testing at $233 \mathrm{~s}^{-1}$ was conducted on a high-speed servo hydraulic machine. A slack adaptor system was employed which ensured that the actuator was able to reach the desired velocity before loading of the sample initiated. Strain measurements were obtained using strain gauges. The test specimens used had gauge 

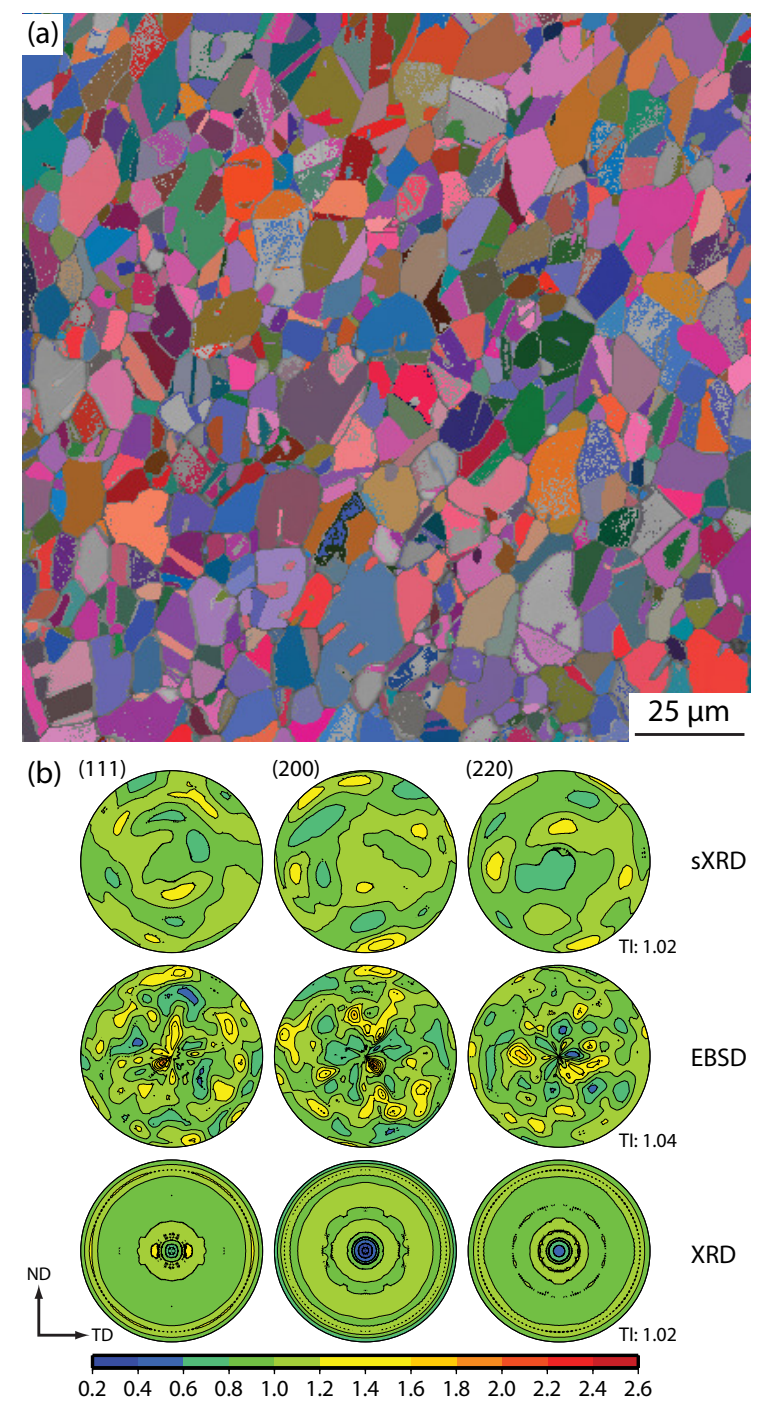

Figure 1: Microstructure and texture including the texture index20 (TI) of the as-received material (a) electron backscatter diffraction ${ }_{201}$ (EBSD) micrograph showing an average grain size of $10 \pm 6 \mu \mathrm{m}$ and (b) random texture determined using synchrotron X-ray diffraction, ${ }^{202}$ EBSD and lab X-ray. EBSD indexing rate of $87 \%$ using a $100 \mathrm{~nm}$ step size.

dimensions of $8 \times 3 \times 2 \mathrm{~mm}$ and were tested along the $\mathrm{RD}_{204}$ of the sample.

\subsection{Hopkinson Pressure Bar and Blast Testing}

A Hopkinson pressure bar was used for the high strain208 rate testing at strain rates of $950 \mathrm{~s}^{-1}$ and above, using209 the same specimen geometry utilised for the intermediate210 strain rate testing, Figure 2(a). The specimen was screwed into the force input and output bars using bespoke grips ${ }^{211}$ which were designed to accommodate the flat specimens, ${ }_{212}$ Figure 2(b). During the testing, an elastic stress pulse is213 introduced into the input bar by the impact of a steel pro-214 jectile on a steel disc attached to one end of the input bar.215 The strain rate the specimen experiences is controlled, to216 some extent, by the impact velocity of the projectile on ${ }_{217}$ the disc and by the gauge length of the specimen. The 218 impact generated elastic stress pulse propagates along the input bar. At the interface between the input bar and the specimen part of the stress pulse is reflected and part passes into the specimen. Similarly, at the interface between the specimen and the output bar part of the stress pulse is reflected, while the remainder is transmitted into the output bar.

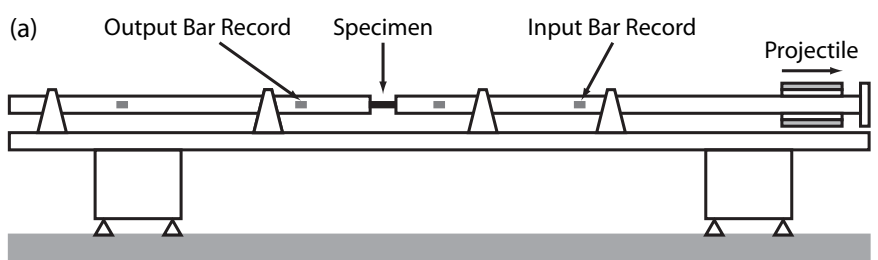

(b)

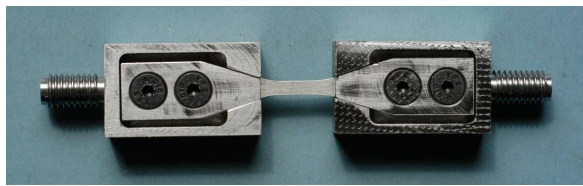

Figure 2: (a) Schematic representation of the Hopkinson pressure bar experimental setup and (b) bespoke clamps used to test flat specimens.

To accurately record the high strain in each specimen during testing, high elongation strain gauges were bonded to the specimen surface. The samples were also painted with speckle paint and digital image correlation (DIC) techniques were employed. High-speed photographic images of the tests, taken typically at rates between 125,000 and 200,000 frames per second were used to measure strain. Finally to accurately record the final failure strain a travelling microscope was used to measure the reduction in area of the sample.

Since the transmitted elastic wave provides a direct measure of the force $(F)$ experienced by the specimen, force was calculated using strain gauges on the output bar and the following relation:

$$
F=E_{\text {out }} \varepsilon_{0} A_{\text {out }}
$$

where $E_{\text {out }}$ is the modulus of the material used for the output bar, $\varepsilon_{0}$ is the strain and $A_{\text {out }}$ is the cross-sectional area of the output bar.

The Hopkinson pressure bar testing was conducted by BAE Systems, Bristol, UK.

Blast testing was conducted on an $800 \times 800 \mathrm{~mm}$ plate using a $160 \mathrm{~mm}$ diameter charge cylinder. Testing was conducted by DSTL, Porton Down, UK.

\subsection{Microscopy}

Samples for light microscopy (LM) and electron backscatter diffraction (EBSD) were prepared following a standard metallographic schedule. Specimens for LM were etched using a solution of $4 \%$ Nital to reveal the grain boundaries.

Backscattered imaging of the twins and electron backscatter diffraction (EBSD) was performed on a Zeiss Auriga FEGSEM fitted with an Oxford Instruments HKL Nordlys 
EBSD detector. Transmission electron microscopy (TEM) analysis to obtain high resolution lattice images was conducted on an FEI Titan 80/300 TEM/STEM microscope, fitted with a monochromator and image aberration corrector. Samples were electropolished using 5 vol.\% perchloric acid and 95 vol.\% acetic acid at $30 \mathrm{~V}$ DC and at room temperature.

\section{Results and Discussion}

\subsection{Mechanical characterisation}

The loading of a specimen in a Hopkinson pressure bar test relies on a stress pulse being generated which is produced by the impact of a projectile on the input bar. Consequently, this limits the test duration which was $\sim 500 \mu \mathrm{s}$ in this investigation. Hence, at a strain rate of $1000 \mathrm{~s}^{-1}$ the final strain in the sample will be $\sim 50 \%$ engineering strain. Consequently, in some instances samples did not fail during the first loading test and thus were reloaded under the same test conditions to induce sample failure.

The dynamic stress-strain response of the TWIP steel subjected to deformation at a range of strain rates can be seen in Figure 3. Here the data presented from the high strain rate Hopkinson pressure bar tests represents the initial loading for each specimen. The specimen tested at a strain rate of $1934 \mathrm{~s}^{-1}$ failed during the first loading. However, specimens tested at strain rates of 1473 and $_{260}$ $1606 \mathrm{~s}^{-1}$ were reloaded to induce failure. Two specimens ${ }^{260}$ were tested to $\sim 40 \%$ true strain at strain rates of $950^{261}$ and $1440 \mathrm{~s}^{-1}$ to investigate the effect of strain rate on the microstructure. Table 1 summarises the testing schedule.

Table 1: Summary of the testing conducted on the TWIP steel where26 some specimens did not fail (DNF) after initial loading and were 267 subsequently reloaded to induce failure while other samples were ${ }_{268}$ tested to similar amount of deformation.

\begin{tabular}{|c|c|c|}
\hline $\begin{array}{l}\text { Strain Rate } \\
\qquad\left(\mathrm{s}^{-1}\right)\end{array}$ & $\begin{array}{c}\text { True Failure Strain }\left(\varepsilon_{t f a i l}\right) \\
(\%)\end{array}$ & Comments \\
\hline 0.001 & 48 & - \\
\hline 0.1 & 54 & - \\
\hline 233 & 53 & - \\
\hline 950 & - & $\begin{array}{c}\text { DNF, } \\
\text { tested to } 38 \% \varepsilon_{t}\end{array}$ \\
\hline 1440 & 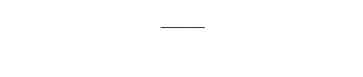 & $\begin{array}{c}\text { DNF, } \\
\text { tested to } 40 \% \varepsilon_{t}\end{array}$ \\
\hline 1473 & 48 & Reloaded \\
\hline 1606 & 47 & Reloaded \\
\hline 1934 & 50 & - \\
\hline
\end{tabular}

Figure 3 reveals a distinct increase in the flow stress ${ }_{28}$ of the material with increasing strain rate. Analysis of ${ }_{282}$ Hopkinson bar data relies on stress equilibrium being at- ${ }_{283}$ tained along the length of the specimen. This typically ${ }_{284}$ necessitates a minimum of three transits of the stress pulse ${ }_{285}$ along the specimen which occurs after $\sim 10 \mu$ s in the cur- $_{286}$ rent investigation. Consequently, at a nominal strain rate $_{287}$ of $950 \mathrm{~s}^{-1}$ data obtained up to $1 \%$ strain must be treated ${ }_{288}$ with caution. However, in spite of this consideration, it $_{289}$

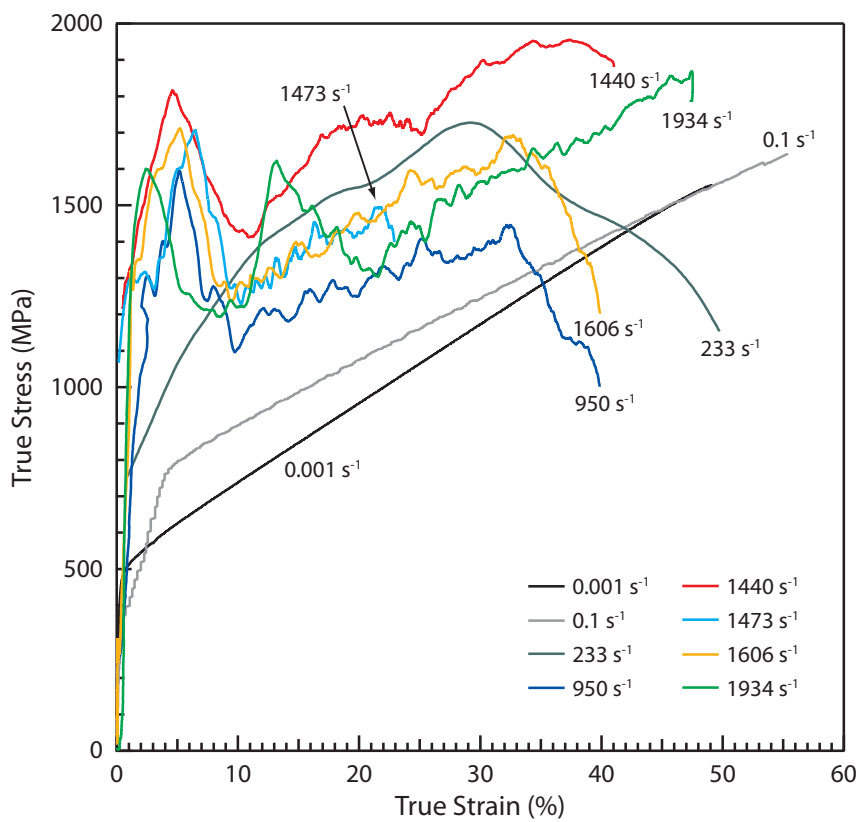

Figure 3: Stress-strain behaviour of the investigated TWIP steel subjected to deformation at a range of strain rates.

appears, from Figure 3, that the investigated material exhibits an upper yield point at higher strain rates since this behaviour is consistently observed. Similar behaviour has been observed by Hwang et al. [21] and Li et al. [22]. Discontinuous yielding in TWIP steels has generally been thought to be caused by the repetitive ageing and depinning of dislocations due to dynamic strain ageing. This gives rise to Portevin-Le Châtelier (PLC) bands, which are regions of localised plastic deformation associated with discontinuous yielding [23]. Similarly, it has also been suggested that, since the discontinuous yielding is often of observed over a large range of strain rates, it may be due to deformation band propagation [24]. This is because twins are efficient stress concentrators; they may emit full and partial dislocations into neighbouring sites which subsequently promote band formation. Nonetheless, it cannot be categorically concluded that the upper yield observed is not due to a non-equilibrium of the stress pulse during the Hopkinson bar testing.

The yield stress exhibits a positive strain rate sensitivity from quasi-static to higher strain rates with a marked increase exhibited at $950 \mathrm{~s}^{-1}$. However, the yield stress exhibits a weak strain rate sensitivity during testing at even higher strain rates. An increase in the yield stress is commonly observed with high strain rate testing and is usually attributed to viscous drag on the moving dislocations during deformation. Therefore, it may be suggested that the weak sensitivity observed at strain rates greater than $950 \mathrm{~s}^{-1}$ are due to relatively smaller viscous drag effects occurring compared to the significant change encountered between 0.001 and $950 \mathrm{~s}^{-1}$. Xiong et al. [17] have reported similar observations with an additional increase in yield stress at $5000 \mathrm{~s}^{-1}$. 


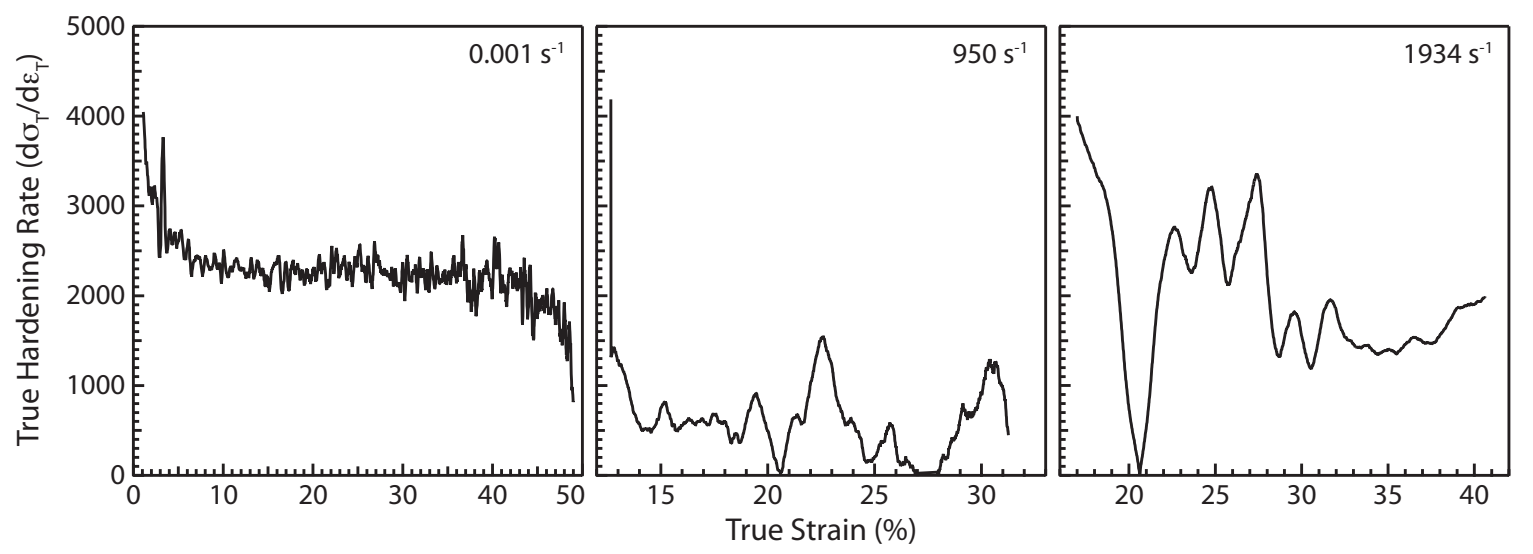

Figure 4: Characteristic hardening behaviour exhibited during deformation at different strain rates.

The total elongation, i.e. failure strain of the material,327 appears to be relatively unaffected by the strain rate, Ta- 328 ble 1. The stress-strain data suggests that twinning may 322 not be hindered with increasing strain rate since a sub-3зо stantial reduction in ductility is not observed during high ${ }_{331}$ rate testing. The increase in the sample temperature due 332 to adiabatic heating during dynamic loading can be esti-3зз mated using the following expression:

$$
\Delta T=\frac{\Delta Q}{\rho C_{p}}=\frac{\beta}{\rho C_{p}} \int_{0}^{\varepsilon_{\max }} \sigma d \varepsilon
$$

where $\Delta Q$ is the fraction of mechanical energy that is con- ${ }^{338}$ verted to heat energy, $\rho$ is the density and $\mathrm{C}_{p}$ is the specific ${ }^{339}$ heat capacity. Assuming that $>90 \%$ of the mechanical en- ${ }^{340}$ ergy is converted to heat (i.e. $\beta=0.9$ ) the temperature ${ }^{341}$ rise at maximum strain during dynamic testing at a strain ${ }^{342}$ rate of $1934 \mathrm{~s}^{-1}$ is estimated to be $\gtrsim 140^{\circ} \mathrm{C}\left(\rho=7.8 \mathrm{gcm}^{-3343}\right.$ and $\left.\mathrm{C}_{p}=0.46 \mathrm{~kJ} / \mathrm{kgK}\right)$. This temperature increase will ${ }^{344}$ consequently produce a significant increase in the stacking fault energy $[3,21]$. However, the relatively insignificant effect of strain rate on the elongation observed during dynamic testing in this study suggests a higher stacking fault energy during the test does not inhibit twinning. Considering the calculated SFE for the experimental material and the associated uncertainty an increase of $\sim 25 \mathrm{~mJ} \mathrm{~m}^{-2}$ in the SFE would still favour deformation twinning. Furthermore, the effect of dynamic loading on twinning is still an area of debate since extensive deformation twinning has been observed during high strain rate testing. In addition, some authors have observed a greater propensity for deformation twinning in materials tested at a high strain rate compared to quasi-static rates [25]. Adiabatic shear banding will also contribute to the overall deformation at higher strain rates.

The strain hardening behaviour of the material exhibits little change with deformation at increasing strain rates, Figure 4. The material exhibits a characteristic high work $_{345}$ hardening rate which is often observed in steels which deformation via twinning. Initially a decrease in the harden- ${ }^{346}$ ing rate with the onset of straining occurs which is followed ${ }_{348}^{347}$ by an increase, although this increase appears to be more significant at the highest strain rate of $1934 \mathrm{~s}^{-1}$. This rise is associated with an increase in the deformation twinning activity and also the possible activation of secondary twin systems. Finally, at higher strain the hardening rate decreases which is due to a reduction in the twinning activity. Although the hardening behaviour observed through the strain rate window is similar, the hardening rate exhibited at $950 \mathrm{~s}^{-1}$ appears to be lower compared to the materials tested at quasi-static and higher strain rates. Hence this may suggest that strain rate has a weak effect on the strain induced hardening mechanism.

The TWIP steel exhibited considerable toughness after blast testing and revealed no indication of bursting under blast loading, Figure 5. Post blast testing a crater with a bulge depth of $\sim 160 \mathrm{~mm}$ was produced along with severe folding of the plate at approximately quarterly intervals around the bulge.

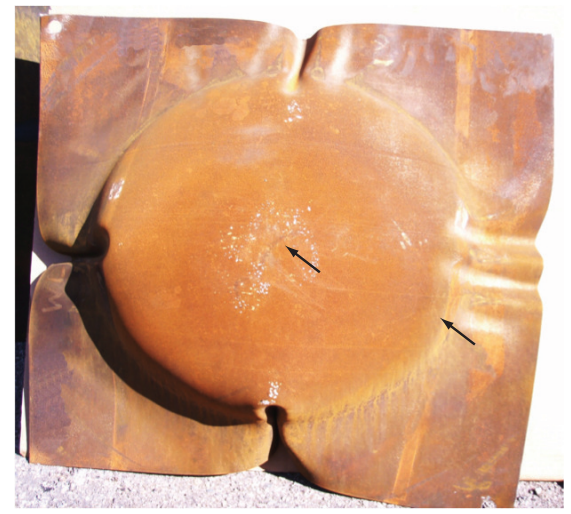

Figure 5: Deformed TWIP steel plate post blast testing. Arrows denote regions from which material was obtained for microstructure characterisation.

\subsection{Microstructure observations}

The final deformed microstructure of the samples tested to failure is shown in Figure 6. The observed microstructure reveals the presence of numerous adiabatic shear bands 

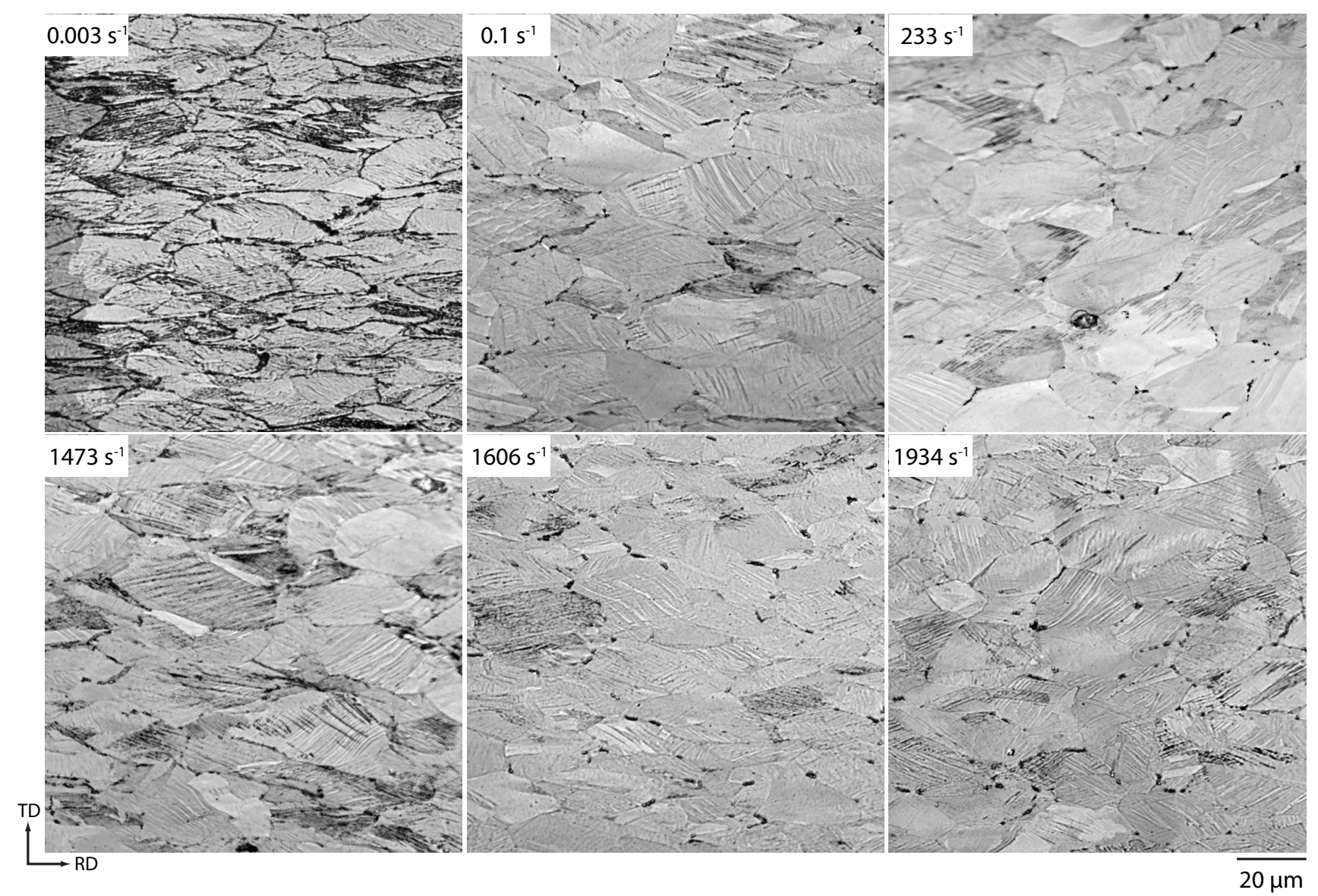

Figure 6: Light microscopy showing the effect of strain rate on the microstructure of the TWIP steel tested to failure, RD = loading direction.

which appear as white bands at $\sim 45^{\circ}$ to the loading di-374 rection (RD). However, this feature is not observed at the 375 slowest strain rate i.e. $0.003 \mathrm{~s}^{-1}$. The presence of shear 376 bands at the higher strain rates demonstrates that adia-377 batic shear localisation is an important deformation mech-378 anism at higher strain rates in addition to slip and twin-379 ning. The origin of adiabatic shear banding is often at-380 tributed to changes within the material at a fundamental 381 level where dislocation pile-up avalanches occur. These 382 are associated with strong microstructural obstacle col-383 lapses [13]. In addition, the deformed microstructures re-384 veal that deformation twinning occurs within the material 385 at all the strain rates tested. However, the density of de-386 formation twins and the number of active twin systems 387 appears to decrease with increasing strain rate, Figure 7. 388

It can be seen from Figure 7 that deformation twin-389 ning is more profuse at the slower strain rate of $233 \mathrm{~s}^{-1} 390$ compared to a strain rate of $1934 \mathrm{~s}^{-1}$. An explanation 391 for this behaviour is most likely do be due to a localised 392 temperature increase within the material caused by adia-393 batic heating. The increment in sample temperature at 394 the experimental strain rates would be sufficient to in-395 crease the stacking fault energy of the material $[\mathbf{3}, \mathbf{1 4}] .396$ This will subsequently reduce the propensity for deforma-397 tion twinning within the material. Furthermore, this phe--398 nomenon has been observed elsewhere in TWIP steels [3, $21,26]$. The deformation twins formed within the sample tested at $233 \mathrm{~s}^{-1}$ appear to be thinner compared to the twins present within the microstructure of the material tested at $1934 \mathrm{~s}^{-1}$. Since deformation twinning is a phenomenon which proceeds through a dislocation mechanism, i.e. the dissociation of a perfect dislocation into partials [10-12], the difference in twin morphology and volume fraction at different strain rates may be due to a dislocation based process. At high strain rates, dislocations will have less time to wait at obstacles to gain sufficient thermal energy to overcome an obstruction. In addition, viscous drag effects also occur. Twin bundles are observed in both samples. However, the bundles formed at the higher strain rate are thicker. A high stacking fault energy also promotes the formation of thicker twins in f.c.c. metals [27], therefore the increase in local sample temperature due to high strain rate deformation may also result in the thicker twins observed at higher strain rates.

Secondary twins are also observed in the material tested at $233 \mathrm{~s}^{-1}$, Figure 7(b). These twins develop within the interspaces of the primary twins and are blocked by them thus forming a 'ladder-like' structure. This phenomenon has been observed elsewhere under quasi-static conditions [28, 

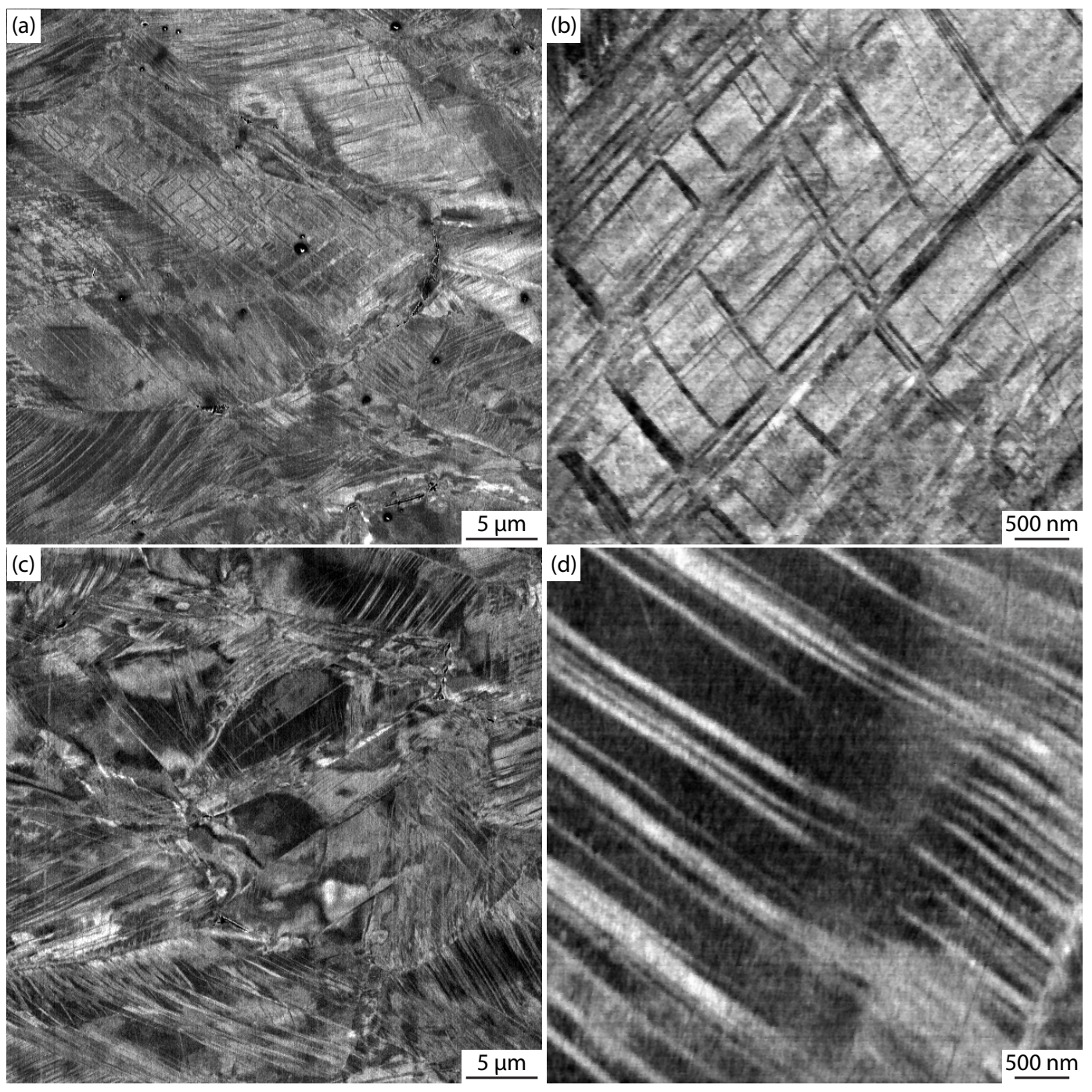

Figure 7: Backscatter electron images demonstrating the effect of strain rate on the twinning behaviour of the TWIP steel tested at (a, b) $233 \mathrm{~s}^{-1}$ and $(\mathrm{c}, \mathrm{d}) 1934 \mathrm{~s}^{-1}$.

29]. The formation of secondary twins at the lower strain 418 rate suggests greater dislocation activity occurring since 419 partial dislocations are required to nucleate and thicken ${ }_{420}$ the twins [10-12]. Li et al. [22] have reported similar ob-421 servations. The width between the twin interspaces also422 appears to be affected by the strain rate, here we observe 423 that the distance between twins is shorter at a high strain ${ }_{424}$ rate. The presence of secondary twin systems and differ-425 ence in twin morphology at a lower strain rate will have 426 an impact on the effect the strain hardening behaviour of ${ }_{427}$ the material. This is evident from the stress-strain be-428 haviour, Figure 3, where a clear difference in the $\operatorname{strain}_{429}$ hardening behaviour at $233 \mathrm{~s}^{-1}$ compared to $1934 \mathrm{~s}^{-1}$ can 430 be observed. The changes in the hardening rate observed ${ }_{431}$ are often associated with the initiation of profuse twinning ${ }_{432}$ and also the activation of new twin systems, which is ob-433 served at $\sim 10 \%$ strain when testing at $233 \mathrm{~s}^{-1}$, Figure 3.434

The effect of strain rate on the deformation behaviour 435 of samples subjected to a similar level of deformation i.e.436 $\sim 50 \%$ strain is shown in Figure 8. The EBSD maps have been reconstructed using inverse pole figure (IPF) colouring relative to the loading direction (rolling direction). This allows grain boundaries to be discerned and orientation relationships within grains to be identified and analysed. A material commonly reacts to deformation through intragranular lattice rotations. This is a manifestation of the plastic strain where dislocation recovery has taken place. Consequently leading to the formation of dislocation subcells. Thus, if these subcells are sufficiently large to be within the spatial resolution of the combined SEM and EBSD systems, minute distortions in the orientation can be visualised. This allows subsequent analysis to be conducted in a quantitative manner. The amount of rotation between the neighbouring pixels within the map will be dependent on the step size utilised in the data acquisition, in addition to intragranular rotations taking place within the material. However, the cumulation rotations can be obtained. 

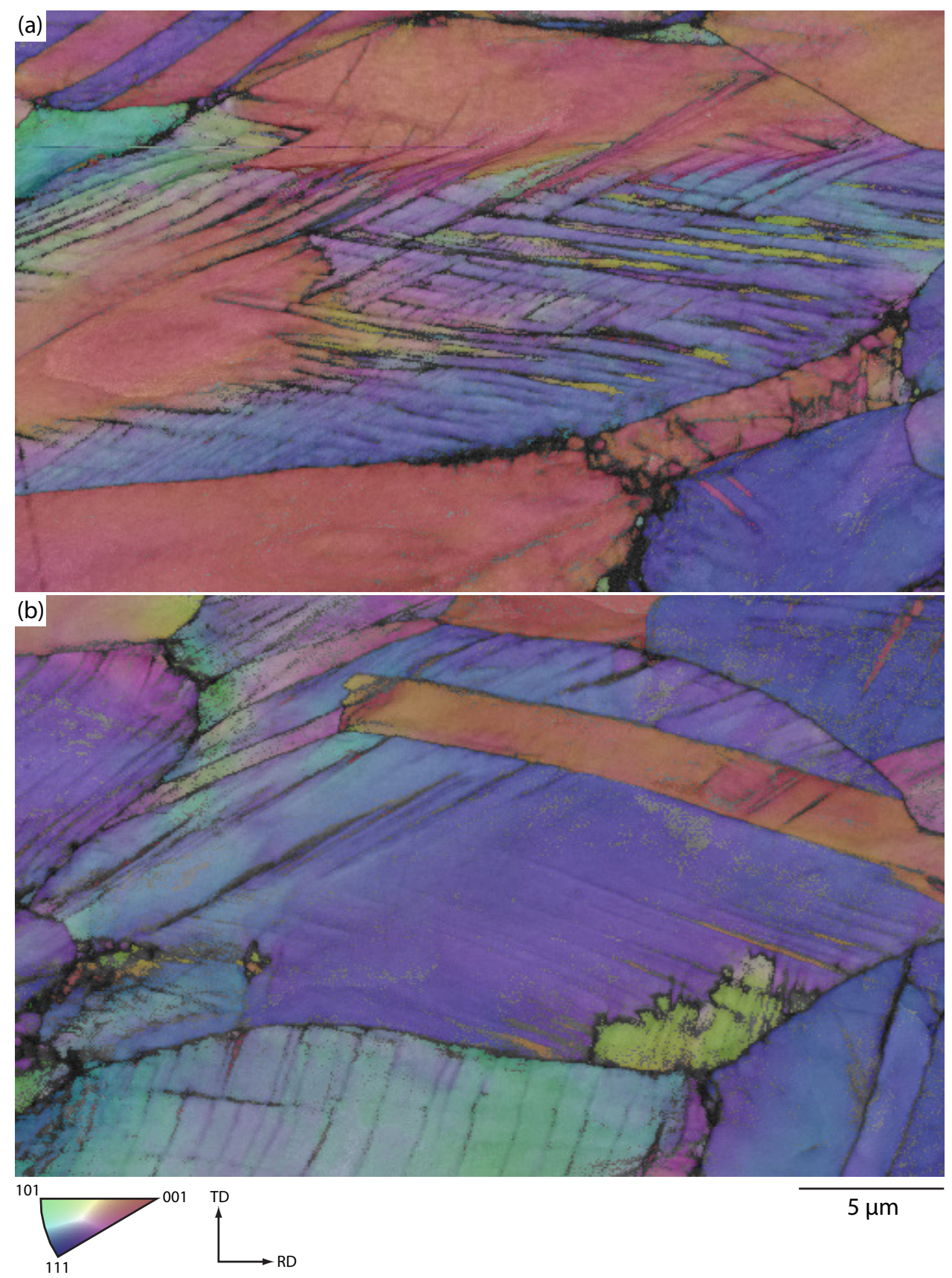

Figure 8: EBSD map with IPF colouring relative to the loading direction (RD) and KPQ colouring showing the effect of strain rate on material tested to approximately $50 \%$ strain at (a) $950 \mathrm{~s}^{-1}$ and (b) $1440 \mathrm{~s}^{-1}$. Indexing rate of $89 \%$ and $85 \%$ respectively using a $50 \mathrm{~nm}$ step size, unindexed points are black.

A large degree of intragranular misorientation is ob-447 served in both samples. However the material tested at 448 $950 \mathrm{~s}^{-1}$ exhibits a larger cumulative level of misorientation 449 i.e. $\sim 25^{\circ}$ over a $10 \mu \mathrm{m}$ distance compared to $\sim 10^{\circ}$ for the 450 material tested at $1440 \mathrm{~s}^{-1}$. Although the relative point 451 to point misorientation in both materials remains small,452 varying between $1-1.5^{\circ}$. The high level of misorientation ${ }_{453}$ observed at the lower strain rate suggests a higher disloca-454 tion density within the grain. Furthermore, both sam-455 ples exhibit a tendency to nucleate twins preferen- 456 tially in grains which have a $\langle\mathbf{1 1 1}\rangle / / \mathbf{R D}$ orientation which is the favoured orientation for deformation twinning in f.c.c. materials that are subjected to tensile loading (i.e. $\mathrm{RD}=$ tensile axis). However, Figure 8 also indicates that profuse twinning is favoured at a lower strain rate for a similar level of deformation. Here we observe the activation of multiple twin systems compared to the sample tested at a higher strain rate of $1440 \mathrm{~s}^{-1}$. The higher level of intragranular misorientation observed at a lower strain rate and the implied higher dis- 
location density would promote the nucleation and growth ${ }_{514}$ of deformation twins. In addition, highly misorientated ${ }_{515}$ subregions are observed within a single grain in the ma-516 terial tested at $950 \mathrm{~s}^{-1}$ which consequently fragment the 517 grain, Figure 8(a). These regions appear to also delimit518 the deformation twin types present within the grain. The 519 band contrast i.e. Kikuchi pattern quality (KPQ) map,520 however, does not suggest the presence of sub-boundaries521 as observed by Barbier et al. [30].

The microstructure of the material subjected to blast523 testing was characterised from two locations, namely from ${ }_{52}$ the centre and wall of the crater created post blast testing,525 denoted by the arrows in Figure 5 . The strain at the centre 526 of the plate was estimated to be $\sim 25 \%$ from the reduction ${ }_{527}$ in the plate thickness post testing. However, the material 528 at the wall of the bulge was subjected to a shear type 529 loading, consequently making an estimate of the strains30 difficult.

Light microscopy reveals relatively few deformation twiss present in the microstructure from both locations, Fig-533 ure 9. Furthermore, adiabatic shear bands which are of-534 ten observed during high strain rate deformation are not535 present while the grain structure appears relatively unde-536 formed. The lack of shear bands may suggest that localised 537 temperature gradients during testing were not sufficient538 to facilitate the formation of the shear bands since the 539 plate tested was relatively large. An interesting feature is 5540 observed in the microstructure of the sample taken from541 the centre of the plate. Here the presence of numerous542 grains which exhibit a 'wavy' characteristic is observed,543 Figure $9(\mathrm{~b}) \&(\mathrm{c})$. X-ray diffraction analysis of the mate-544 rial using a slow scan i.e. $1000 \mathrm{~s}$ count time per point,545 does not reveal the presence of any additional phases other than austenite, such as $\varepsilon$-martenite, Figure 10. Furthermore, similar grains are not observed in the microstructure around the wall of the crater. Similar microstructures are occasionally observed in f.c.c. materials which are subjected to an increasing pre-strain on the twinning plane. This results in the spacing between twinned regions to increase and causes the twin boundaries to become wavy. This occurrence may also be due to a strong interaction between initial twins and slip [31]. Since the loading on the material during blast testing is complex and not a simple tension mechanism, it is possible for twins to form early in certain orientations which are subsequently rotated with further deformation. This rotation is accompanied by a strong interaction with slip thus resulting in the observed wavy microstructure.

Electron backscatter diffraction (EBSD) of specimens taken from the centre and wall of the blast crater reveal the relative lack to deformation twins at the wall, Figure 11. However, EBSD does indicate the formation of numerous twins in the specimen taken from the centre of the blast crater, Figure 11(a). An interesting observation here is that the twins form in grains which are orientated along the line between the $\langle 001\rangle / / \mathrm{BA}$ (blast axis) and $\langle 111\rangle / / \mathrm{BA}$, Figure 11(c). Analogous to slip, twinning proceeds according to its Schmid factor distribution. During uniaxial tension grains with $\langle 111\rangle$ near parallel to the tensile axis exhibit Schmid factors for twinning which are higher to that for slip. Consequently, these grains exhibit a large volume fraction of twins and also contain multiple twin systems. Conversely, during compression twinning is favoured in grains which are orientated with their $\langle 001\rangle$ parallel to the loading axis, since the Schmid factor for twinning is higher compared to slip during compression within such grains. The distribution of the majority of deformation twins along $\langle 001\rangle / / \mathrm{BA}$ and $\langle 111\rangle / / \mathrm{BA}$ is most likely due to the complex loading on the material during blast testing. The Schmid factor of the grains within which deformation twinning is observed in Figure 11(a) was calculated using the Channel 5 EBSD software. The results revealed that grains in the $\langle 111\rangle / / \mathrm{BA}$ orientation which also contained twins have a relatively larger Schmid factor value for twinning compared to slip. However, grains containing twins with an orientation lying along the $\langle 001\rangle / / \mathrm{BA}$ and $\langle 111\rangle / / \mathrm{BA}$ line had either Schmid factors for twinning which were almost identical to that for slip or they exhibited a higher Schmid factor for twinning than that for slip. Furthermore, the calculated twin Schmid factors for these grains were also higher than the twin Schmid factor for grains in the $\langle 111\rangle / / \mathrm{BA}$ orientation. This indicates that during blast loading the formation of deformation twins is relatively easy in grains lying along the $\langle 001\rangle / / \mathrm{BA}$ and $\langle 111\rangle / / \mathrm{BA}$ line. In addition, a number of the deformation twin bundles identified in Figure 11(a) are evidently thicker than $100 \mathrm{~nm}$ since they are easily indexed using the resolution of the EBSD technique. This suggests that twinning is relatively pro-

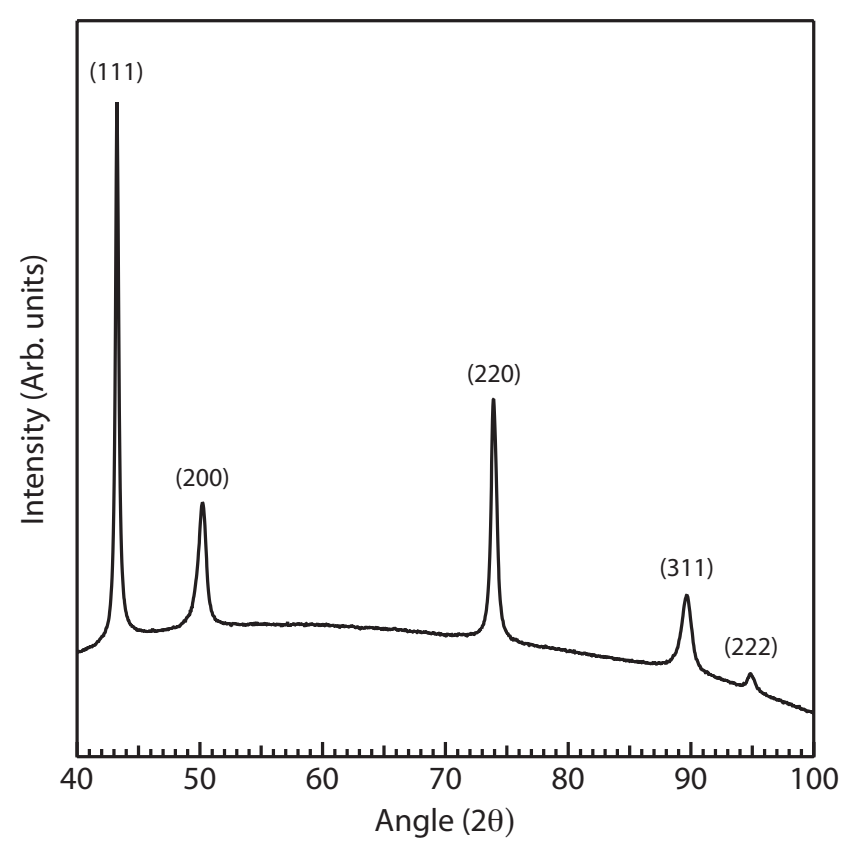

Figure 10: XRD scan of material taken from the centre of the blast plate does not indicate the presence of secondary phases such as $\varepsilon$-martenite. 

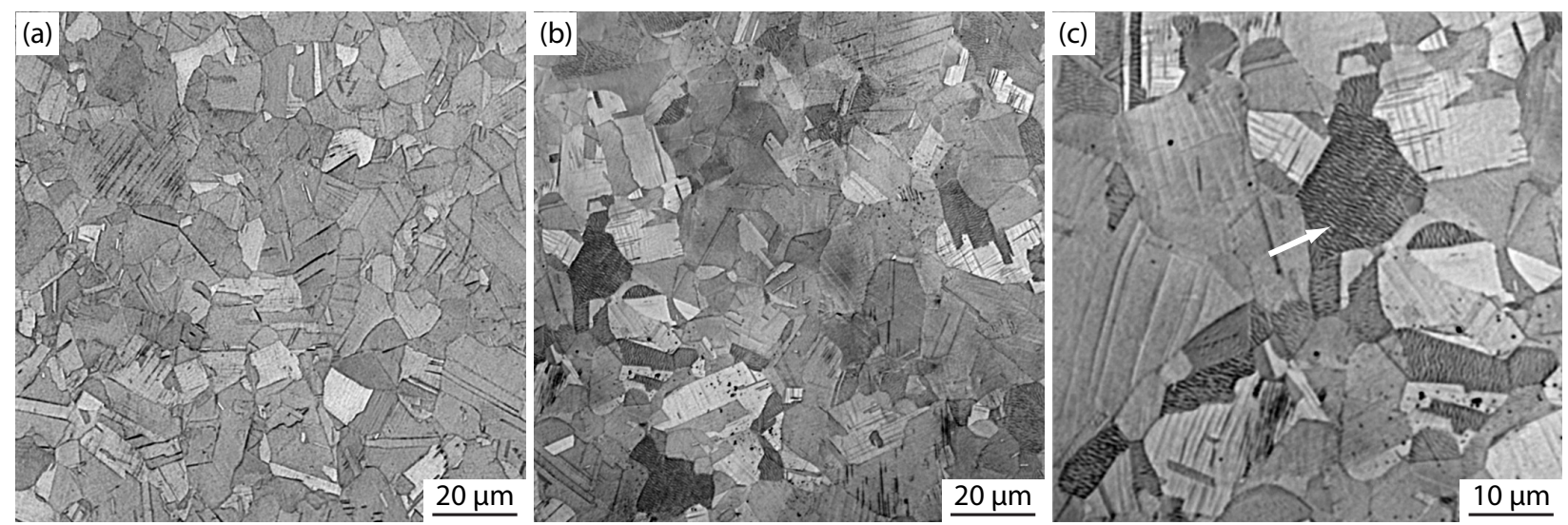

Figure 9: Microstructure of the steel plate post blast testing taken from (a) the wall of the crater created during testing and (b, c) the centre of the plate which also exhibits numerous grains with a 'wavy' characteristic (denoted by the arrow).

fuse in these orientations since the twin bundle thickness584 is large.

Transmission electron microscopy of the blasted mate- ${ }^{585}$ rial examined from the centre of the blast crater revealed ${ }^{586}$ numerous deformation twins, Figure 12. The structure ${ }^{587}$ around and within the twins indicated the presence of $n u^{-58}$ merous stacking faults. The cell type structure that $\operatorname{can}^{589}$ be seen in Figure 12(e) is produced by a high density of ${ }^{50}$ stacking faults. The faults that were examined within the $e^{591}$ twins during our analysis were determined to be intrinsic ${ }^{592}$ stacking faults. Multiple stacking faults within a range ${ }^{593}$ of a few atomic layers were also observed as indicated in ${ }^{594}$ Figure 12(d). The internal twin structure was imaged on ${ }^{595}$ the [110] zone axis. In addition, Figure 12(d) was post596 processed to observe the faults more clearly. Firstly $a^{597}$ band-pass (or top-hat) filter was applied to the Fourier ${ }^{598}$ transform pseudo-diffraction image. Then a deconvolution ${ }^{599}$ process using a Gaussian kernel was applied to the image. .000 Finally a reconvolution process was applied using a smaller601 size kernel. Even though the high resolution images are fil-602 tered it is difficult to clearly visualise some defects, such ${ }^{603}$ as two faults in close proximity to each other.

The apparent presence of only intrinsic stacking faults ${ }^{605}$ is consistent with observations made by Idrissi et al. [32]600 where intrinsic stacking faults were observed in high man-607 ganese steels that deform via twinning and extrinsic faults ${ }^{608}$ were observed when the $\varepsilon$ martensite transformation oc-609 curs. A structure similar to the 'wavy' grains observed ${ }^{610}$ under light microscopy (Figure 9) was also observed us-611 ing TEM, Figure 12(f). This was observed in some grains, ${ }^{612}$ while neighbouring grains did not exhibit a similar struc-613 ture. Finally, it should be noted that phase contrast imag-614 ing is sensitive to strain. Therefore it becomes difficult to ${ }^{615}$ analyse high resolution TEM images. Hence it is suggested ${ }^{616}$ that further work needs to be conducted to elucidate the ${ }^{617}$ current observations using high resolution, high angle an-618 nular dark field (HAADF) STEM and centre of symmetry ${ }^{619}$ analysis [33, 34].

\section{Conclusions}

The effect of dynamic strain rates on the mechanical behaviour and microstructure of a TWIP steel have been investigated using Hopkinson pressure bar testing and blast testing. The mechanical response of the material was augmented through microstructural characterisation using a range of techniques including electron backscatter diffraction (EBSD). Subsequently, the following conclusions can be drawn from the investigation:

1. A $15 \mathrm{Mn}-2 \mathrm{Al}-2 \mathrm{Si}-0.7 \mathrm{C}$ wt. $\%$ TWIP steel was tested at a range of strain rates between $10^{-3}-1934 \mathrm{~s}^{-1}$ using Hopkinson pressure bar and blast testing.

2. A distinct increase in the flow stress of the material was observed at higher strain rates. The yield stress exhibited a positive strain rate sensitivity from quasistatic strain rates up to $950 \mathrm{~s}^{-1}$, however, only a weak sensitivity is observed at even higher strain rates.

3. Strain rate appeared to have a weak influence on the failure strain of the investigated material. Furthermore, the characteristic hardening behaviour also remained similar with increasing strain rate.

4. The investigated TWIP steel exhibited considerable ductility during blast testing.

5. The microstructure of the material indicated that deformation twinning was less profuse at higher strain rates, while all the samples tested exhibited adiabatic shear bands apart from the material loaded at a strain rate of $0.003 \mathrm{~s}^{-1}$. In addition, adiabatic shear bands did not form during blast testing.

6. EBSD revealed the formation of multiple twin systems during deformation at lower strain rates which was not observed at higher strain rate. At lower strain rates, the material exhibited larger intragranular misorientations suggesting larger dislocations densities existing in the material.

7. The microstructure of the blast tested specimen exhibited a 'wavy' microstructure in selected grains, which is likely to have developed due to the complex loading experienced during testing. 

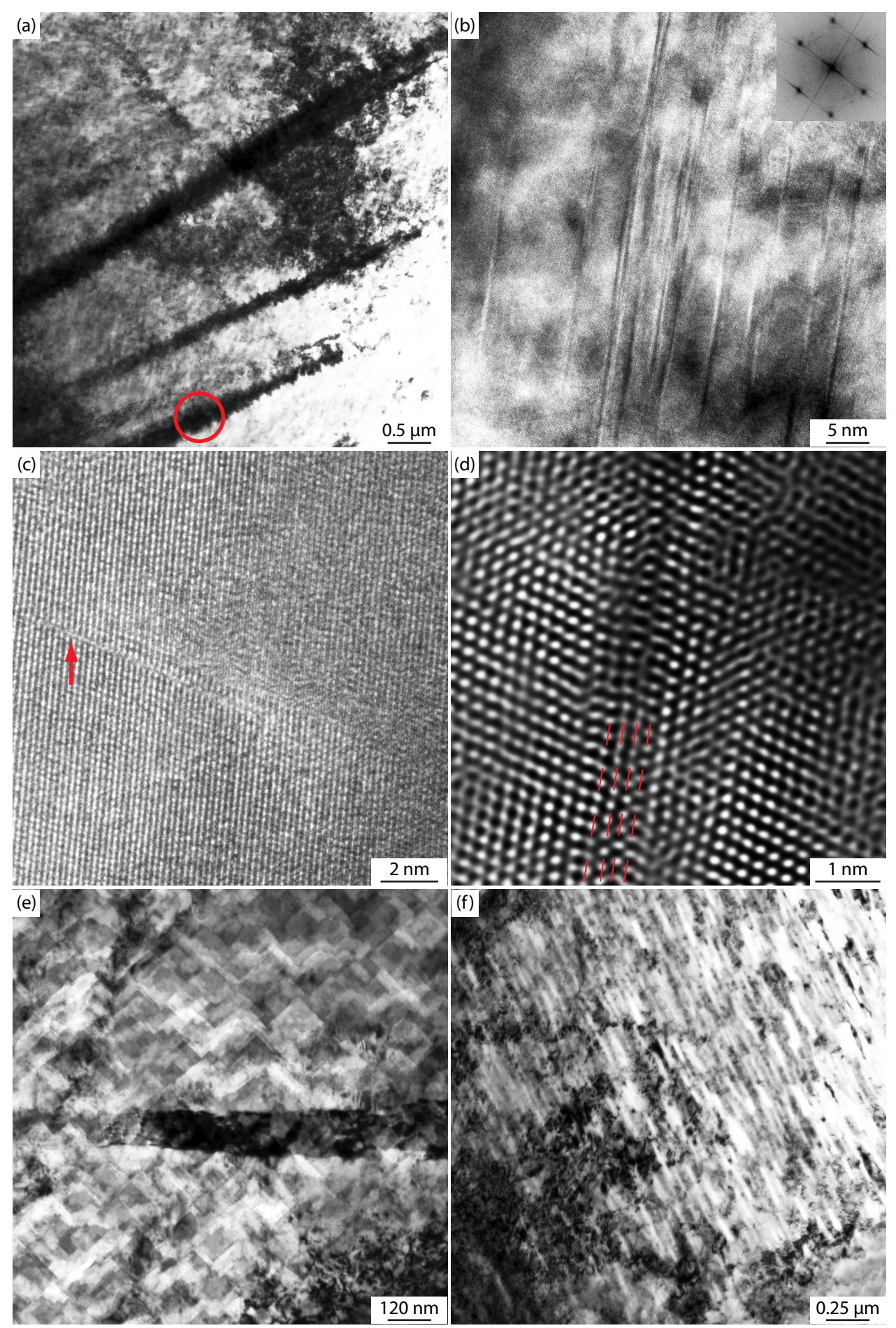

Figure 12: High resolution transmission electron microscopy of blasted material examined from the centre of the blast crater revealing (a) numerous deformation twins, (b-d) internal structure of a selected twin (circled) taken on the [110] zone axis, showing numerous intrinsic stacking faults (arrow), (e) high density of stacking faults around the twin and (f) wavy microstructure similar to that observed under light microscopy. 


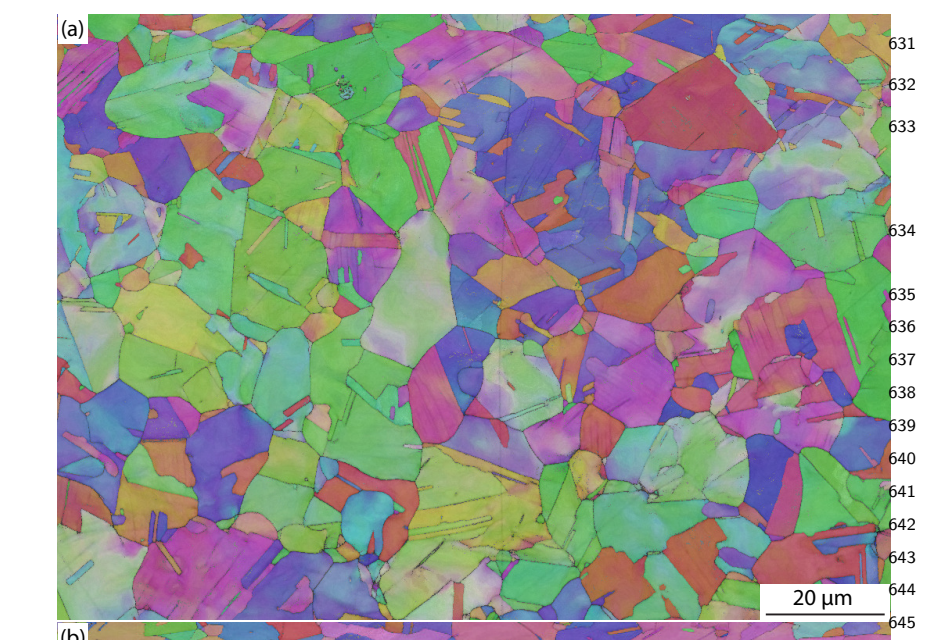

from the materials and structures research programme delivered by Team MAST for the Defence Technology and Innovation Centre, part of the UK Ministry of Defence.

\section{References}

[1] G Frommeyer and O Grassel. Material Science and Technology, 14 (1998) 1213-1216

[2] G Frommeyer, U Brux, and P Neumann. ISIJ International 43 (2003) 438-446

[3] S Curtze and V-T Kuokkala. Acta Materialia, 58 (2010) 51295141

[4] S Allain, J.P Chateau, O Bouaziz, S Migot, and N Guelton. Materials Science and Engineering A 387 (2004) 158-162

[5] O Bouaziz, S Allain, C P Scott, P Cugy, and D Barbier. Current Opinion in Solid State \& Materials Science 15 (2011) 141-168

[6] BW Oh, SJ Cho, YG Kim, YP Kim, WS Kim, and SH Hong. Materials Science and Engineering A, 197 (1995) 147-156

[7] PCJ Gallagher. Metallurgical Transactions 1 (1970) 2429-2461

[8] R Schramm and R Reed. Metallurgical and Materials Transactions A 6 (1975) 1345-1351

[9] H Idrissi, K Renard, L Ryelandt, D Schryvers, and PJ Jacques. Acta Materialia 58 (2010) 2464-2476

[10] J. A. Venables. Philosophical Magazine 30 (1974) 1165-1169

[11] J. B. Cohen and J Weertman. Acta Metallurgica 11 (1963) 996998

[12] S Mahajan and GY Chin. Acta Metallurgica 21 (1973) 13531363

[13] R W Armstrong and S M Walley. International Materials Reviews 53 (2008) 105-128

[14] G F Bolling and R H Richman. Acta Metallurgica 18 (1970) $673-681$

[15] J.W Christian and S Mahajan. Progress in Materials Science, 39 (1995) 1-157

[16] G F Bolling and R H Richman. Acta Metallurgica 13 (1965) 723-743

[17] Zhi. Ping. Xiong, Xue. Ping. Ren, Wei. Ping. Bao, Shu. Xia. $\mathrm{Li}$, and Hai. Tao. Qu. Materials Science and Engineering A 530 (2011) 426-431

[18] Puspendu Sahu, Sven Curtze, Arpan Das, B Mahato, VeliTapani Kuokkala, and Sandip Ghosh Chowdhury. Scripta Materialia 62 (2010) 5-8

[19] N Li, Y D Wang, R Lin Peng, X Sun, P K Liaw, G L Wu, L Wang, and H N Cai. Acta Materialia 59 (2011) 6369-6377

[20] W Bleck, K Phiu-on, C Heering, and G Hirt. Steel Research International 78 (2007) 536-545

[21] Si Woo Hwang, Jung Hoon Ji, and Kyung-Tae Park. Materials Science and Engineering A 528 (2011) 7267-7275

Figure 11: EBSD maps with IPF colouring relative to the blast $\mathrm{di}^{676}$ rection (ND) coupled with band contrast showing material response ${ }_{678}^{677}$ at (a) the bulge centre, (b) bulge edge when subjected to blast load- ${ }_{679}^{678}$ ing. Indexing rate of $97 \%$ and $98 \%$ respectively using a step size ${ }_{680}^{679}$ of $100 \mathrm{~nm}$. IPF map showing the orientations in which deformation ${ }_{68}^{68}$ twins are observed corresponding to the microstructure observed at ${ }_{682}^{681}$ the centre of the blast crater(c).

[22] D Li, Y Wei, C Liu, L Hou, D Liu, and X Jin. Journal of Iron and Steel Research International 17 (2010) 67-73

[23] P Zavattieri, V Savic, L Hector, J Fekete, W Tong, and Y Xuan. International Journal of Plasticity 25 (2009) 2298-2330

[24] M A Lebyodkin, T A Lebedkina, A Roth, and S Allain. Acta Physica Polonica A 122 (2012) 478-481

[25] O Grassel, L Kruger, G Frommeyer, and L.W Meyer. International Journal of Plasticity 16 (2000) 1391-1409

8. TEM revealed the presence of a high density of intrin-686 sic stacking faults both around and within the twins687 in the blasted material.

\section{Acknowledgements}

The authors would like to thank PF Morris, M Cor- ${ }_{694}^{693}$ nelissen, PA Davies and B Berkhout from Tata steel and695 PM Brown from DSTL, UK for their support in useful dis- ${ }^{696}$ cussions and for material supply. This work was supported ${ }_{698}^{697}$
[26] M Hokka, V.-T Kuokkala, S Curtze, T Vuoristo, and M Apostol. J. Phys. IV France 134 (2006) 1301-1306

[27] J. F. M. Vergnol, J. R. Grilhe Journal de Physique 45 (1984) $1479-1490$

[28] S Allain, J Chateau, D Dahmoun, and O Bouaziz. Materials Science and Engineering A 387 (2004) 272-276

[29] JA Jiménez and G Frommeyer. Materials Characterisation 61 (2010) 221-226

[30] D Barbier, N Gey, S Allain, N Bozzolo, and M Humbert. Materials Science and Engineering A 500 (2009) 196-206

[31] L Meng, P Yang, Q Xie, H Ding, and Z Tang. Scripta Materialia 56 (2007) 931-934

[32] H. Idrissi, L. Ryelandt, M. Veron, D. Schryvers, and P. J. Jacques. Scripta Materialia 60 (2009) 941-944 
[33] V. A. Vorontsov, L. Kovarik, M. J. Mills, and C. M. F. Rae. Acta Materialia 60 (2012) 4866-4878

[34] L. Kovarik, R. Unocic, J. Li, P. Sarosi, C. Shen, Y. Wang, and M. Mills. Progress in Materials Science 54 (2009) 839-873 